\title{
ESTUDIO DE LA INTERCALACIÓN DE POLIANILINA EN MINERALES DE ARCILLA PARA FORMAR MATERIALES MICRO-COMPUESTOS
}

\section{STUDYING THE INTERCALATION OF POLYANILINE IN CLAY MINERALS TO MAKE MICRO-COMPOSITES MATERIALS}

\author{
J. G. Carriazo*11, J. A. Torres ${ }^{2}$, A. E. Ensuncho ${ }^{3}$
}

${ }^{1}$ Dr. Sc.-Química. Departamento de Química, Facultad de Ciencias, Universidad Nacional de Colombia. Ciudad Universitaria, Carrera 30 No. 45-03. Bogotá, D.C. E-mail: jcarriazog@unal.edu.co; ${ }^{2}$ Químico, Departamento de Química, Facultad de Ciencias, Universidad Nacional de Colombia. Ciudad Universitaria, Carrera 30 No. 45-03. Bogotá, D.C. E-mail: jatorreslu@ unal.edu.co. ${ }^{3}$ M.Sc.- Química. Departamento de Química, Facultad de Ciencias e Ingeniería, Universidad de Córdoba. Montería-Colombia. E-mail: aensuncho@sinu.unicordoba.edu.co.

Rev. U.D.CA Act. \& Div. Cient. 15(2): 481 - 489, 2012

\section{RESUMEN}

El presente trabajo muestra el estudio de la intercalación de especies de polianilina en dos minerales de arcilla para formar materiales micro-compuestos, con propiedades en el transporte de electrones. Los sólidos fueron preparados mediante la síntesis "in situ" de polianilina en matrices, de dos arcillas colombianas tipo montmorillonita. Los materiales fueron evaluados por microscopía electrónica de barrido (SEM), difracción de rayos X (DRX) y medidas de resistencia eléctrica. Los resultados de SEM destacan la morfología granular e irregular de los materiales compuestos y la DRX verifica intercalación y delaminación. Las medidas de resistencia eléctrica permitieron confirmar la potencialidad de algunos materiales como sólidos conductores de corriente y proporcionaron información adicional sobre la posible distribución y configuración de las fases componentes de los materiales compuestos de polianilina/montmorillonita.

Palabras clave: Material compuesto, nanocompuesto, polianilina, arcilla.

\section{SUMMARY}

This paper reports a study of the polyaniline-species intercalation into two clay minerals to form micro-composite materials with properties for electron transport. The solids were prepared by "in situ" synthesis of polyaniline into the matrix of two Colombian montmorillonite-clay types. The materials were evaluated by scanning electron microscopy (SEM), X-ray diffraction (XRD) and electrical resistance measurements. An irregular grain morphology for composite materials was observed by SEM, and the intercalated/ delaminated structures were verified by XRD analyses. The potential performance of some of the solids to be current conductors was confirmed by electrical resistance measurements, which also gave additional information on the possible distribution and configuration of the constituting phases of polyaniline/montmorillonite composites.

Key words: Composite material, nanocomposite, polyaniline, clay.

\section{INTRODUCCIÓN}

En los últimos años es notable el avance creciente en el diseño de nuevos materiales que, finalmente, logran ser incorporados de manera exitosa en los procesos de manufactura involucrados en las cadenas productivas de alta tecnología (Dobrzanski, 2006). En esta vía, se ha logrado obtener materiales poliméricos con propiedades conductoras, semiconductoras, con propiedades importantes de óxido-reducción $\mathrm{y}$, algunas veces, con propiedades anticorrosivas (Lu et al. 2011; Dai et al. 2010; Bajpai et al. 2006; Campbell, 2002; Min, 1999), en todos los casos, con múltiples aplicaciones industriales.

Aunque los polímeros conductores poseen enormes ventajas por sus propiedades en diferentes eventos que implican el transporte de electrones, al tiempo que conservan características de materiales plásticos: bajo costo, flexibilidad, facilidad de sintetizar y de moldear, entre otras, es evidente su desventaja en términos de resistencia mecánica y térmica (Ayatollahi et al. 2011; Dai et al. 2010; Bur et al. 2005). En consecuencia, para mejorar estas propiedades se han generado diversos procedimientos que conducen 
a la preparación de "materiales compuestos", resultantes de la incorporación controlada de materiales cerámicos apropiados, como dióxido de titanio, nanotubos de carbono y aluminosilicatos, entre otros (Su \& Gan, 2012; Ayatollahi et al. 2011; Deka \& Kumar, 2011; Ballav \& Biswas, 2006).

Por definición, un material compuesto es una mezcla de materiales diferentes que aportan sus propiedades individuales para potencializar ciertas características deseadas en el sólido final (Smith \& Hashemi, 2006). Desde este punto de vista, el uso de minerales de arcilla, preferiblemente, los conocidos como montmorillonitas o esmectitas, son bastante utilizados, como matrices cerámicas, en la conformación de materiales compuestos, complementados con fases poliméricas. Múltiples publicaciones indican las ventajas estructurales en el diseño de materiales compuestos basados en polímeros con minerales de arcilla (Malwela \& Ray, 2011; Deka \& Kumar, 2010; Franco \& Maspoch, 2009; Rodríguez et al. 2008; do Nascimento et al. 2006; Yeh et al. 2002). La incorporación de montmorillonitas en sistemas poliméricos permite fortalecer la resistencia mecánica, mejorar la resistencia térmica y controlar la conductividad eléctrica de los plásticos iniciales (Deka \& Kumar, 2010; Franco \& Maspoch, 2009).

Las esmectitas o montmorillonitas son aluminosilicatos laminares con estructura tipo T:O:T o 2:1 (Figura 1a), formados por redes octaédricas, en las que un catión $\mathrm{Al}^{3+}$ está rodeado por seis grupos $\mathrm{OH}$ o átomos de oxígeno, en medio de dos láminas tetraédricas de $\mathrm{SiO}_{4}$ (Carriazo et al. 2007). Entre las láminas T:O:T, se encuentran cationes de compensación $\left(\mathrm{Na}^{+}, \mathrm{Ca}^{2+}\right)$, que pueden ser intercambiados para lograr la inserción de especies voluminosas, que permiten incrementar, apreciablemente, el espaciado basal $\mathrm{d}_{001}$ original (Carriazo et al. 2009).

Variadas fases poliméricas se han empleado en el diseño de materiales tipo micro y nano-compuestos, dependiendo de las propiedades deseadas y las aplicaciones requeridas. Una estructura polimérica empleada con frecuencia por sus propiedades conductoras de electricidad es la polianilina (Bekri-Abbes \& Srasra, 2010; Narayanan et al. 2010; Rodríguez et al. 2008; do Nascimento et al. 2006; Yeh et al. 2001), un polímero orgánico conjugado, producto de la polimerización oxidativa de la anilina en medio ácido que, generalmente, presenta conductividad, debido a la deslocalización de carga en su estructura y de acuerdo a su nivel de oxidación (Figura 1b).

El objetivo del presente trabajo es obtener información complementaria sobre la organización estructural real de los componentes de un conjunto de materiales micro-
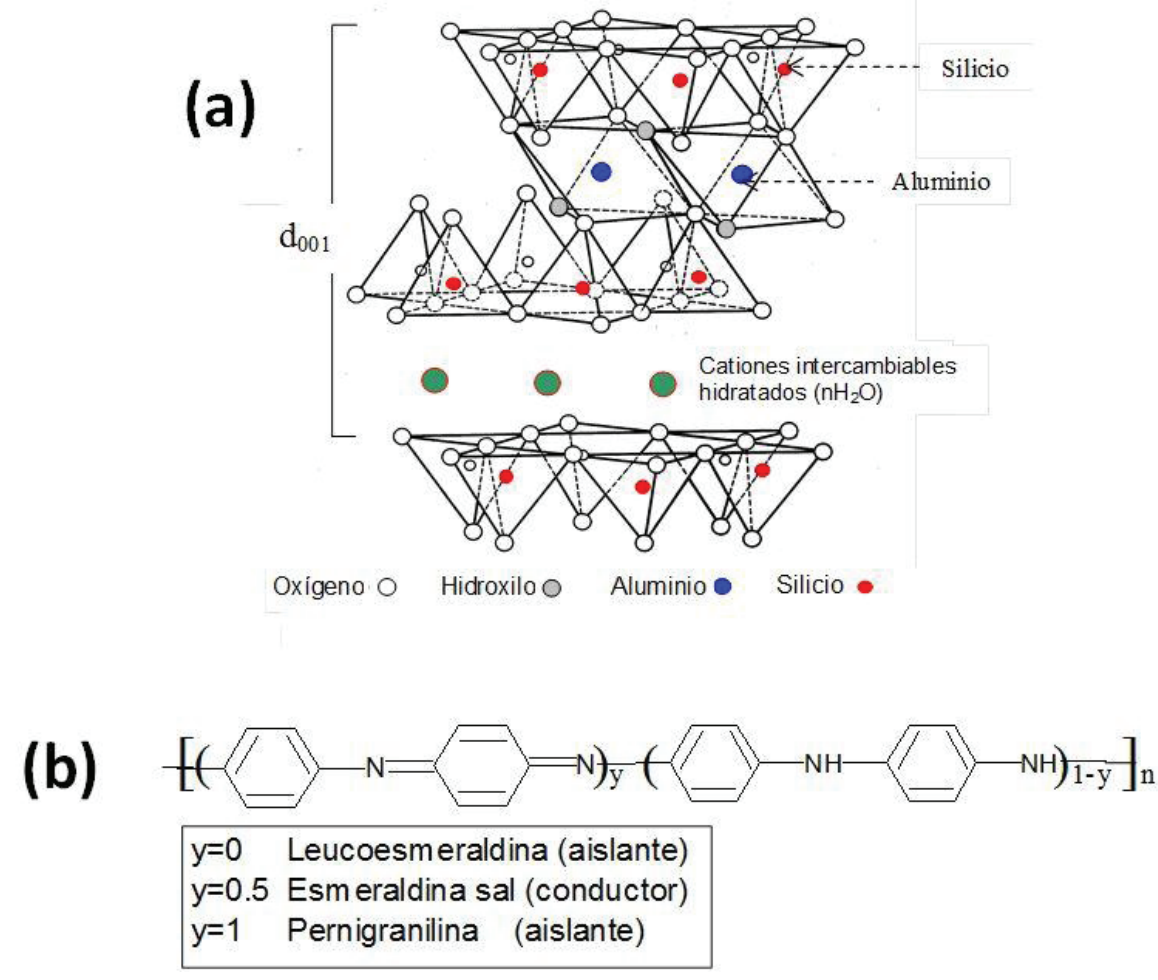

Figura 1. a) Estructura de un mineral de arcilla 2:1 (esmectita); b) Estructura general de una cadena de polianilina para tres niveles de oxidación (tres formas del polímero). 
compuestos de polianilina/montmorillonita, con la participación de dos arcillas explotadas en Colombia, cuya preparación ya ha sido publicada (Rodríguez et al. 2008) y cuyo interés radica en sus propiedades en el transporte de electrones. Este trabajo involucra la verificación de un modelo de organización de las fases componentes de los materiales, lo que permitirá avanzar en la optimización de estos sólidos, para futuras aplicaciones en sistemas de alta tecnología.

\section{MATERIALES Y MÉTODOS}

La síntesis de los sólidos, se encuentra descrita de manera detallada en un trabajo anterior (Rodríguez et al. 2008). Para ello, se emplearon dos arcillas tipo esmectita: una bentonita del Valle del Cauca (BVC) y una montmorillonita proveniente del centro del país, denominada en adelante M64. Ambas arcillas, se saturaron previamente coniones $\mathrm{Na}^{+}$, obteniéndose los sólidos BVC/Na y M64/Na, para luego, proceder con la síntesis de la polianilina en el interior de las láminas de cada mineral, vía intercalación con iones alquilamonio, a partir de una sal de amonio cuaternaria (bromuro de hexadeciltrimetilamonio $\left.\left(\mathrm{CH}_{3}\left(\mathrm{CH}_{2}\right)_{15} \mathrm{~N}\left(\mathrm{CH}_{3}\right)_{3} \mathrm{Br}\right)\right)$, como se describe en literatura (Rodríguez et al. 2008).

Las arcillas intercaladas con cationes alquilamonio (BVC/ Alqm y M64/Alqm), se sometieron a procedimientos posteriores de adición de anililna y persulfato de amonio, para obtener el polímero (polianilina) intercalado en los minerales, en dos formas principales: esmeraldina protonada (PANI-H), conocida como esmeraldina sal (conductora de electricidad) y esmeraldina base (PANI-B, no conductora de electricidad). Los materiales compuestos que se obtuvieron, se denotan como BVC/PANI-H, BVC/PANI-B, M64/PANI-H y M64/PANI-B.
Las imágenes de microscopía electrónica de barrido (SEM), se tomaron en un microscopio FEI QUANTA 200 y los difractogramas de rayos $\mathrm{X}$, en un equipo $\mathrm{X}$ Pert Pro MPD PANalitical, con ánodo de Cu (radiación Ka, $\lambda=1,54056 \AA ̊$ ), mediante la técnica de polvo forzado, a temperatura ambiente, con tamaño de paso de $0,05^{\circ} 2 \theta$ y tiempo de paso $2 \mathrm{s.}$

Para las medidas de resistencia eléctrica, se construyeron probetas delgadas de $13 \mathrm{~mm}$ de diámetro (Tabla 1 y Figura 2a), mediante compresión de las muestras en un pastillador de acero inoxidable, teniendo control estricto de las masas, en una balanza Sartorius Research R200D, de cinco cifras decimales. Las medidas de diámetro y de altura de las probetas (Tabla 1), se hicieron con un calibrador digital marca DISCOVER ${ }^{\circ}$.

Para medir la resistencia, a través del tiempo, se construyeron electrodos de $\mathrm{Cu}$ de alta pureza (>99,99\%), de diámetro igual a las probetas, sometidos a abrasión y limpieza antes de cada medida y sujetados a la probeta bajo presión. Las medidas, se hicieron en función del tiempo para observar la estabilidad de los materiales frente a posibles fenómenos de oxido-reducción. Los datos continuos de resistencia, se tomaron usando un multímetro UT60E RS232C y un sistema computarizado (software UT60E Interface Program ver 2.02). La temperatura, se registró fijando una termocupla de 1,0 mm de diámetro junto a las muestras. Cada muestra y la termocupla fueron envueltas en lana de vidrio (aislante térmico), para evitar las fugas térmicas.

Adicionalmente, se realizaron medidas sobre probetas, de igual masa y diámetro que las anteriores, compuestas

Tabla 1. Masa y dimensiones de cada una de las probetas diseñadas.

\begin{tabular}{|c|c|c|c|c|}
\hline Sólidos & Masa $(\mathbf{g})$ & Presión (psi) & Diámetro (mm) & Altura (mm) \\
\hline BVC/Na & 0,16333 & 22,0 & 13,0 & 0,64 \\
\hline BVC/Alqm & 0,16330 & 22,0 & 13,0 & 0,79 \\
\hline BVC/PANI-H & 0,16331 & 22,0 & 13,0 & 1,00 \\
\hline BVC/PANI-B & 0,16334 & 22,0 & 13,0 & 0,95 \\
\hline & & & & 0,71 \\
\hline M64/Na & 0,16336 & 22,0 & 13,0 & 0,81 \\
\hline M64/Alqm & 0,16338 & 22,0 & 13,0 & 0,92 \\
\hline M64/PANI-H & 0,16333 & 22,0 & 13,0 & 0,97 \\
\hline M64/PANI-B & 0,16331 & 22,0 & 13,0 & \\
\hline
\end{tabular}




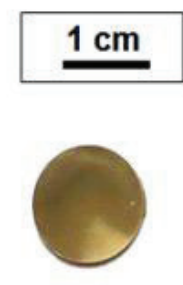

BVC/Alqm
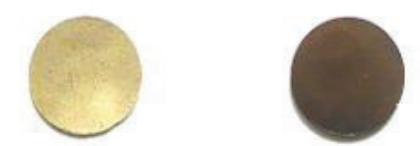

$\mathrm{BVC} / \mathrm{Na}$

BVC/PANI-H

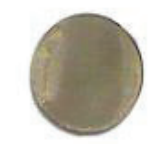

M64/Alqm

(a)

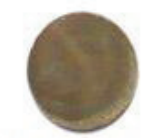

$\mathrm{M} 64 / \mathrm{Na}$
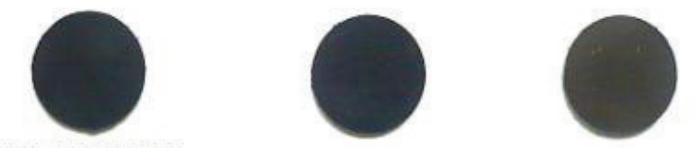

BVC/PANI-B

M64/PANI-B

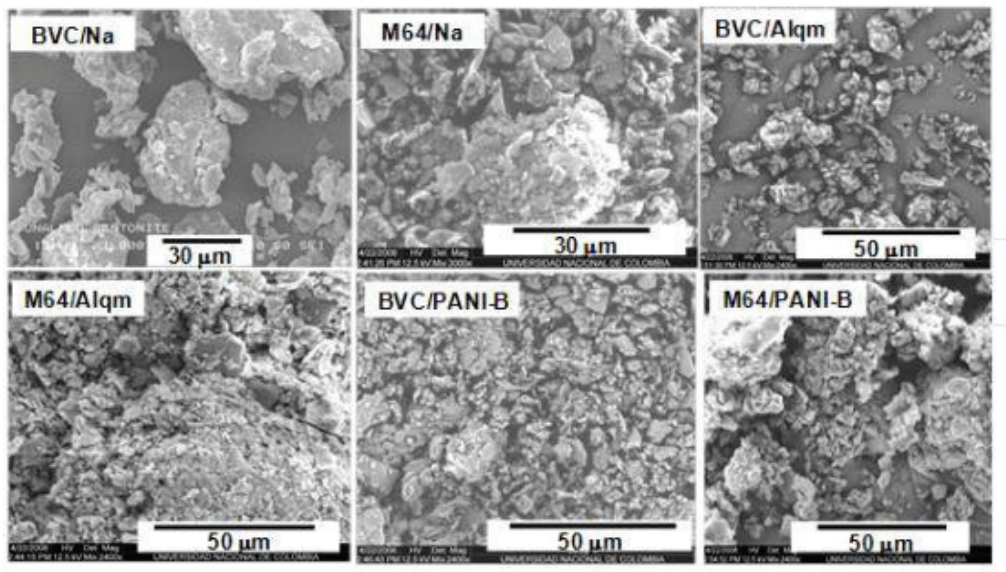

(b)
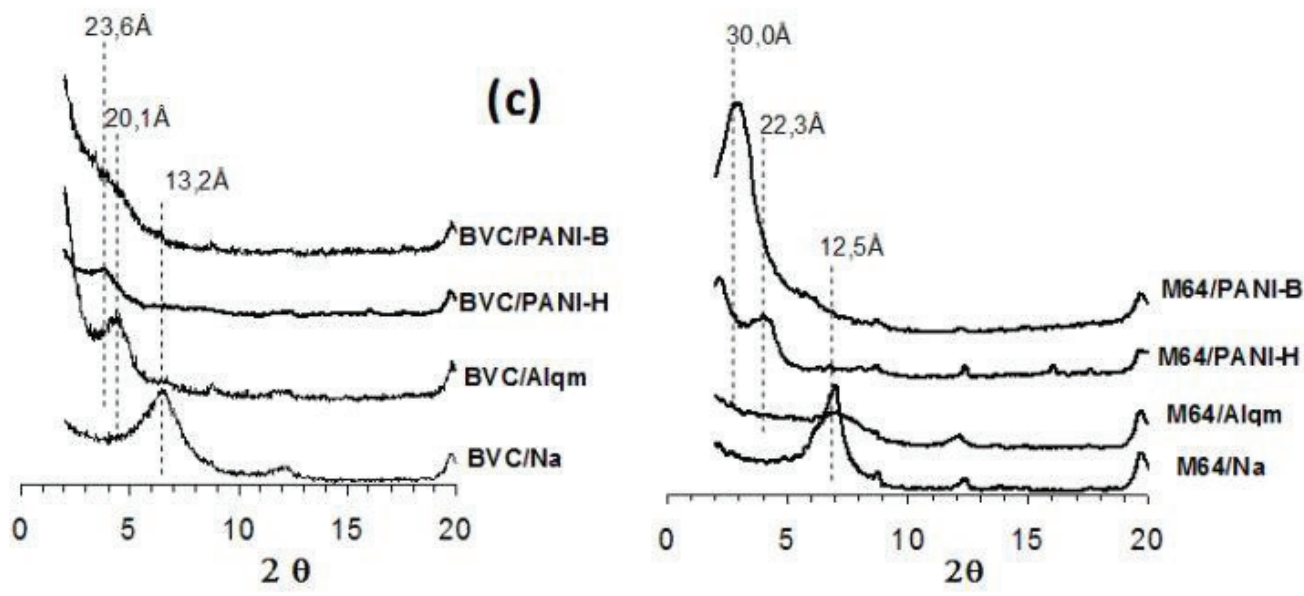

Figura 2. a) Fotografías de las probetas elaboradas con los diferentes materiales para medir sus propiedades eléctricas. b) Imágenes de microscopía electrónica de barrido (SEM) de las muestras en polvo, c) Difractogramas de rayos X de las arcillas naturales y de materiales micro-compuestos. 
de la mezcla de bentonita y polvo de grafito, con el objeto de observar el comportamiento eléctrico del material, con diferentes cargas de un conductor granular reconocido (grafito en polvo). Se obtuvieron probetas con 1\%, 5\%, 7\%, $9 \%$, de grafito en bentonita y $100 \%$, de grafito. En cada caso, se adicionaron las cantidades requeridas y la mezcla (o el material único) se trituró en un mortero de ágata, hasta homogeneizarla.

\section{RESULTADOS Y DISCUSIÓN}

Las imágenes obtenidas por SEM (Figura 2b) confirman la morfología granular e irregular a nivel microscópico en todas las muestras, tanto en las arcillas sódicas como en los materiales compuestos. El material compuesto, se presenta de manera muy discreta, mediante la formación de granos muy pequeños, los cuales, luego de ejercer presión en la construcción de las probetas, deben generar límites de grano o de brechas entre partículas, lo que puede llegar a ser importante al momento de la conducción de electrones.

Los difractogramas obtenidos (Figura 2c) confirman la intercalación de las especies poliméricas sintetizadas, en los espacios interlaminares de los minerales de arcilla. El desplazamiento de la señal correspondiente al espaciado basal $d_{001}$ (con valores de $12,5 \AA$ y 13,2 $\AA$ para M64/Na y $\mathrm{BVC} / \mathrm{Na}$, respectivamente) hacia ángulos menores, es decir, hacia valores mayores de espaciado interlaminar, verifica la introducción efectiva de las especies de polianilina, entre láminas de arcilla. Además, se observa un desplazamiento efectivo de esta señal en el sólido BVC/Alqm y no en M64/ Alqm, lo que indica la posible intercalación de cantidades mayores de los cationes alquilamonio, en la bentonita y una intercalación incipiente, en la arcilla M64.

Evidentemente, este paso tiene fuerte influencia en la síntesis posterior "in situ" de las especies de polianilina, lo que se verifica en los difractogramas de los sólidos M64/PANI-B y BVC/PANI-B. Para el material M64/PANI-B, se observa la señal típica de intercalación con espaciado basal $\mathrm{d}_{001}=30,0$ $\AA$, pero para el sólido BVC/PANI-B, se percibe el perfil de difracción típico de un material delaminado o exfoliado. Este resultado muestra diferencias estructurales en los materiales compuestos obtenidos, lo cual, se origina por las variaciones fisicoquímicas que existen entre los minerales de partida, que se reflejan en su capacidad expansiva.

La figura 3 muestra las medidas de resistencia eléctrica de los materiales estudiados. Los sólidos BVC/PANI-B y M64/ PANI-B no se incluyen, debido a que sus valores de resistencia son muy elevados y superaron los límites de medida. De hecho, la forma de polianilina incorporada en estos sólidos (PANI-B), conocida como esmeraldina base, es no conductora y empleada, frecuentemente, como protectora contra la corrosión en metales. Las arcillas sódicas (VBC/Na y $\mathrm{M64} / \mathrm{Na}$ ) presentan valores de resistencia eléctrica bastante elevados; sin embargo, no son totalmente aislantes, como se esperaba; se observan ciertos valores de conductancia de corriente, como consecuencia de la humedad de estos materiales y la presencia de iones sodio, fundamentalmente.

En general, se observa, como tendencia, la disminución de la resistencia eléctrica, en la medida en que se incorporan las especies poliméricas (PANI-H) o los iones alquilamonio; no obstante, en el caso del sólido M64/Alqm, no hay una disminución considerable de la resistencia eléctrica con respecto a las mediciones sobre el material de partida (M64/ $\mathrm{Na})$.

La diferencia en conductancia (el inverso multiplicativo de la resistencia eléctrica) entre los sólidos M64/Alqm y BVC/Alqm, se puede explicar a partir de la incorporación incipiente de iones alquilamonio en la arcilla M64, lo cual, se verifica en sus perfiles de difracción de rayos X (Figura 2c) y también justifica el comportamiento eléctrico parecido al de su correspondiente arcilla sódica. Adicionalmente, los sólidos tienden a aumentar su resistencia con el tiempo, en especial, para los materiales con mayores valores de resistencia eléctrica, lo que muy probablemente está relacionado con el aumento de temperatura de estas muestras, donde se registraron elevaciones de temperatura hasta de $15^{\circ} \mathrm{C}$, para las arcillas sódicas. Para estos materiales de resistencia elevada, gran parte de la corriente se transforma en calor, lo que genera mayor dificultad para que un conductor eléctrico permita el paso de electrones.

Para verificar las observaciones anteriores, se diseñaron experimentos con un conductor eléctrico reconocido (grafito en polvo) en la matriz arcillosa (bentonita sódica), en diferentes proporciones (Figura 4), encontrándose que, efectivamente, al aumentar las cantidades de grafito en la bentonita, la resistencia de los materiales tiende a cero y en los materiales con menor contenido de grafito ( 1 y $5 \%$ ), la resistencia eléctrica tiende a elevarse con el tiempo, como consecuencia del calentamiento de las muestras.

De otro lado, en todos los materiales intercalados (arcillas con alquilamonio y polianilina conductora), se observaron caídas súbitas en la resistencia eléctrica (Figura 3), indicando una elevación repentina en el paso de corriente, aumento súbito y breve de la conductancia. Este comportamiento sugiere el paso forzado de micro-barreras dieléctricas después de la acumulación de ciertos valores de carga eléctrica y, en conjunto, con los resultados de difracción de rayos X, los cuales, indicaron intercalación o delaminación de las arcillas, permite considerar que, a nivel microscópico, parte de la población de las láminas del mineral de arcilla están desorientadas y obstaculizando la conexión efectiva entre las 

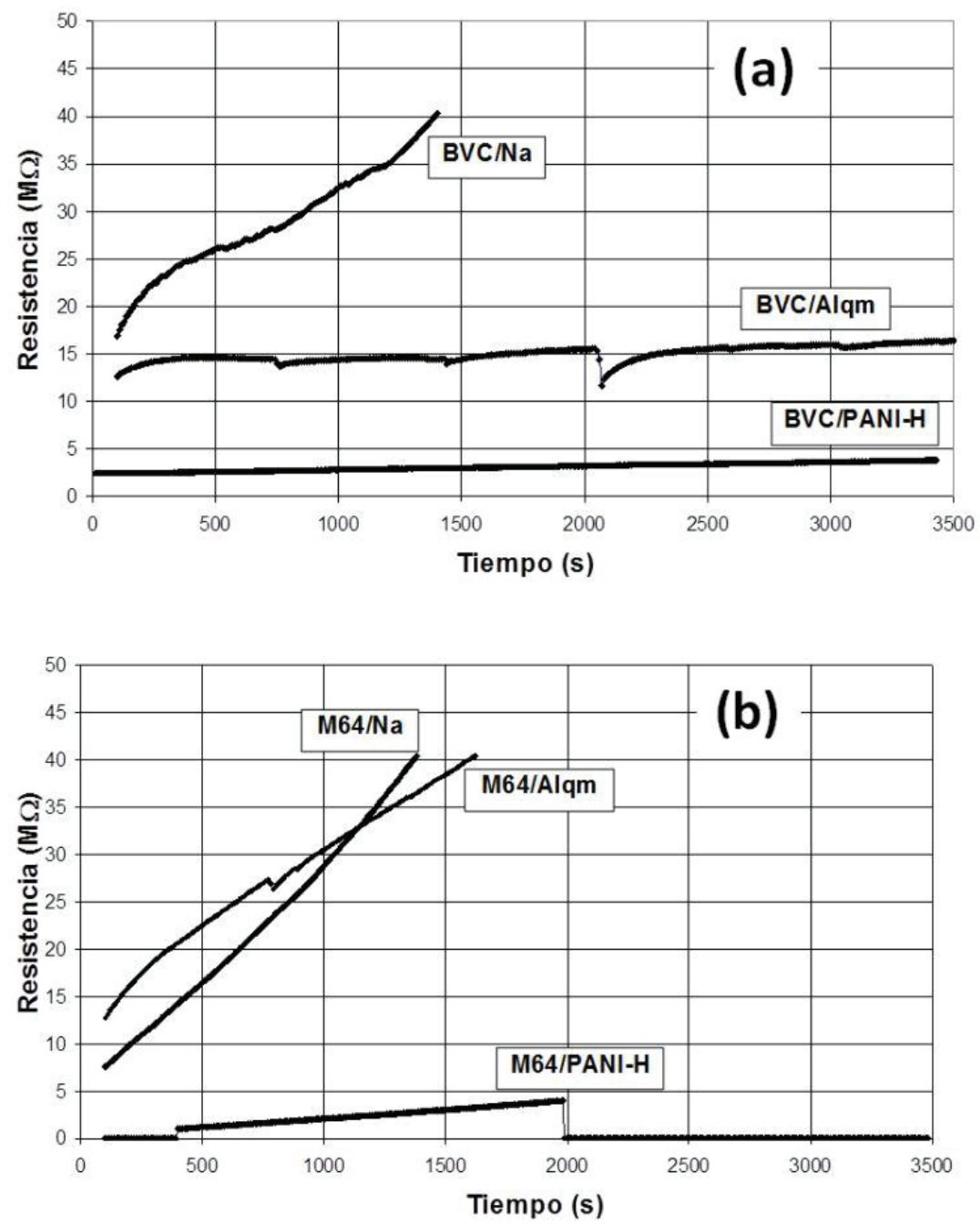

Figura 3. Variación de la resistencia eléctrica con el tiempo, en las arcillas sódicas (BVC/Na y M64/Na), en las arcillas intercaladas con iones alquilamonio (BVC/Alqm y M64/Alqm) y en los materiales compuestos de arcilla con polianilina en su forma conductora (BVC/PANI-H y M64/PANI-H).

macromoléculas de polianilina conductora (Figura 5).

Las figuras $5 \mathrm{a}$ y $5 \mathrm{~b}$ indican la posible disposición de láminas de arcilla que interfieren en el flujo de corriente eléctrica (micro-barreras dieléctricas). Las figuras 5 c y 5 d representan la intercalación ideal (ordenada) de las macromoléculas de polianilina en el mineral de arcilla; sin embargo, un conjunto de apilamientos de láminas ("clay layer stacking"), se puede situar frente a otro con diferente orientación, generando interrupciones de conectividad. Este modelo de intercalación de polianilina conduce a la reflexión sobre nuevos métodos de preparación de este tipo de materiales, en el que, probablemente, será necesario conceder mayor importancia al grado de polimerización de la polianilina, es decir, lograr macromoléculas de mayor tamaño, para favorecer la conectividad entre las mismas, sin el sacrificio excesivo de las propiedades mecánicas de los materiales. Adicionalmente, el modelo enseña la necesidad de recurrir a minerales de arcilla con tamaños de partícula muy pequeños y mineralógicamente bastante puros, toda vez que la presencia de partículas de otros minerales no expandibles podrían estar interfiriendo, tanto en el proceso de síntesis como en el paso de corriente sobre los sólidos.

En conclusión, la intercalación de especies conductoras de polianilina (esmeraldina sal) mediante su síntesis "in situ" en los espacios interlaminares de dos arcillas tipo montmorillonita, de origen mineralógico colombiano, conduce a la obtención de materiales micro-compuestos con propiedades eléctricas muy importantes, convirtiendo a los minerales de partida en sólidos conductores de electricidad, 

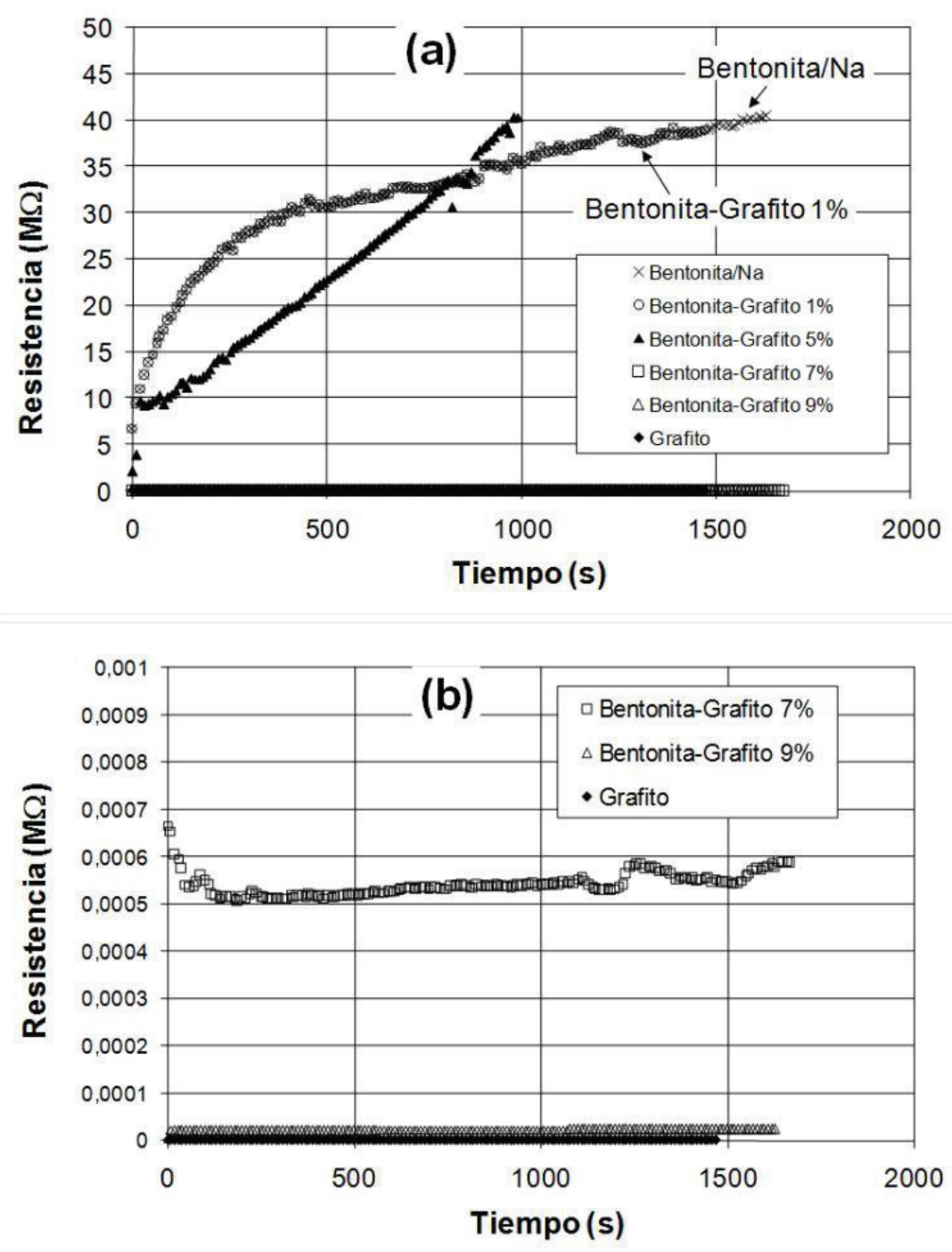

Figura 4. Medidas de resistencia eléctrica para materiales compuestos preparados mediante la mezcla de grafito/bentonita. a) Bentonita sódica, diferentes cargas de grafito y grafito puro; b) Ampliación de la escala para las curvas cercanas a cero.

(a)

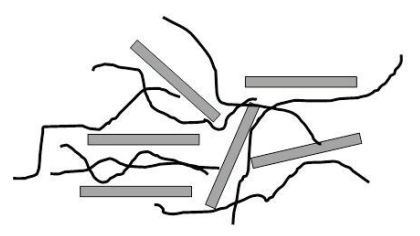

(b)

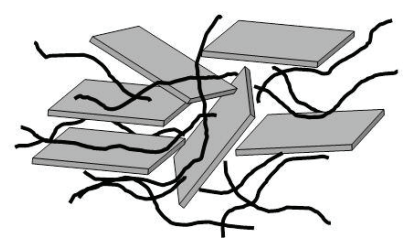

(c)

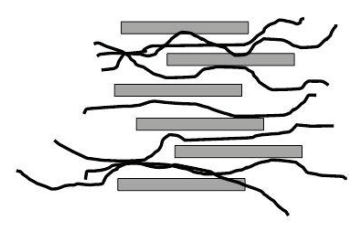

(d)

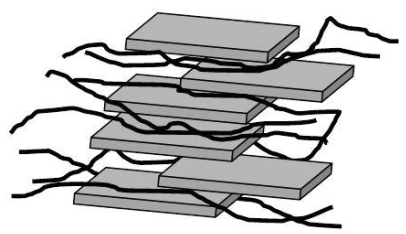

Figura 5. Modelo de la posible organización estructural de los componentes de los materiales micro-compuestos de polianilina/arcilla. a) y c) representan vistas laterales, b) y d) representan vistas en perspectiva. 
con el aporte de las propiedades de matrices cerámicas. Los resultados de difracción de rayos $\mathrm{X}$ confirmaron la intercalación de las especies poliméricas y ponen en evidencia la influencia de la naturaleza mineralógica de las arcillas iniciales en la formación de materiales exfoliados. Las medidas de conducción eléctrica, además de revelar la potencialidad de los sólidos conductores, proporcionaron información adicional sobre la posible distribución y configuración de las fases componentes en los materiales compuestos de polianilina/montmorillonita.

Agradecimientos: Los autores ofrecen sus agradecimientos a la Universidad Nacional de Colombia, por el apoyo logístico. Conflicto de intereses: El presente trabajo fue preparado con la participación de todos los autores, quienes declaramos que no existe conflicto de intereses que ponga en riesgo la validez de los resultados o la voluntad de publicación de los mismos.

\section{BIBLIOGRAFÍA}

1. AYATOLLAHI, M.R.; SHADLOU, S.; SHOKRIEH, M.M.; CHITSAZZADEH, M. 2011. Effect of multi-walled carbon nanotube aspect ratio on mechanical and electrical properties of epoxy-based nanocomposites. Polymer Testing. 30:548-556.

2. BALLAV, N.; BISWAS, M. 2006. Conductive composites of polyaniline and polypyrrole with $\mathrm{MoO}_{3}$. Materials Letters. 60:514-517.

3. BEKRI-ABBES, I.; SRASRA, E. 2010. Characterization and $A C$ conductivity of polyanilinemontmorillonitenanocomposites synthesized by mechanical/chemical reaction. Reactive \& Functional Polymers. 70:11-18.

4. BUR, A.J.; LEE, Y.H.; ROTH, S.C.; START, P.R. 2005. Measuring the extent of exfoliation in polymer/ clay nanocomposites using real-time process monitoring methods. Polymer. 46:10908-10918.

5. BAJPAI, V.; HE, P.; GOETTLER, L.; DONG, J.H.; DAI, L. 2006. Controlled syntheses of conducting polymer micro- and nano-structures for potential applications. Synthetic Metals. 156:466-469.

6. CAMPBELL, D.K. 2002. Conducting polymers and relativistic field theories. Synthetic Metals. 125:117128.

7. CARRIAZO, J.G.; SAAVEDRA, M.J.; MOLINA, M.F. 2009. Estudio por DRX de la intercalación-pilarización de un mineral de arcilla tipo 2:1 con especies polioxocatiónicas de aluminio. Rev. Mex. Ing. Quím. 8(3):299-305.

8. CARRIAZO, J.; MOLINA, R.; MORENO, S. 2007. Caracterización estructural y textural de una bentonita colombiana. Rev. Col. Quím. 36(2):213225.

9. DAI, T.; SHI, Z.; SHEN, C.; WANG, J.; LU Y. 2010. Selfstrengthened conducting polymer hydrogels. Synthetic Metals. 160:1101-1106.

10. DEKA, M.; KUMAR, A. 2011. Electrical and electrochemical studies of poly(vinylidene fluoride)-clay nanocomposite gel polymer electrolytes for Li-ion batteries. J. Power Source. 196:1358-1364.

11. DEKA, M.; KUMAR, A. 2010. Enhanced electrical and electrochemical properties of PMMAclay nanocomposite gel polymer electrolytes. Electrochim. Acta.55:1836-1842.

12. DOBRZANSKI, L.A. 2006. Significance of materials science for the future development of societies. J. Mat. Proces. Techn. 175:133-148.

13. DO NASCIMENTO, G.M.; CONSTANTINO, V.R.L.; LANDERS, R.; TEMPERINI, M.L.A. 2006. Spectroscopic characterization of polyaniline formed in the presence of montmorillonite clay. Polymer. 47:6131-6139.

14. FRANCO, E.A.; MASPOCH, M.L. 2009. Estructura general de las arcillas utilizadas en la preparación de nanocompuestos poliméricos. Ingenierias. 12(44):35-41.

15. LU, X.; ZHANG, W.; WANG, C.; WEN, T.C.; WEI, Y. 2011. One-dimensional conducting polymer nanocomposites: Synthesis, properties and applications. Progr. Polymer Sci. 36:671-712.

16. MALWELA, T.; RAY, S.S. 2011. Unique morphology of dispersed clay particles in a polymer nanocomposite. Polymer. 52:1297-1301.

17. MIN, G. 1999. Conducting polymers and their applications in the film industry. Polyaniline/Polyimide Blended Films. Synthetic Metals. 102:1163-1166.

18. NARAYANAN, B.N.; KOODATHIL, R.; GANGADHARAN, T.; YAAKOB, Z.; SAIDU, F.K.; CHANDRALAYAM, S. 
2010. Preparation and characterization of exfoliated polyaniline/montmorillonite nanocomposites. Mat. Sci. Engineer. B. 168:242-244.

19. RODRÍGUEZ, J.; CARRIAZO, J.; CORREDOR, P.; MOLINA, F.; MORENO, S. 2008. Síntesis de materiales microcompuestos de polianilina/arcilla: caracterización y evaluación de su actividad como agentes anticorrosivos. Rev. Col. Quím. 37(3):337353.

20. SMITH, W.F.; HASHEMI, J. 2006. Fundamentos de la Ciencia e Ingeniería de Materiales. Ed. MacGrawHill (México, D. F.). 1032p.

21. SU, L.; GAN, Y.X. 2012. Experimental study on synthesizing $\mathrm{TiO}_{2}$ nanotube/polyaniline (PANI) nanocomposites and their thermoelectric and photosensitive property characterization. Composites: Part B. 43:170-182.

22. YEH, J-M.; LIOU, S-J.; LIN, C-Y.; CHENG, C-Y.; CHANG, Y-W.; LEE, K-R. 2002. Anticorrosively enhanced PMMA-clay nanocomposite materials with quaternary alkylphosphonium salt as an intercalating agent. Chem. Materials. 14:154-161.

23. YEH, J-M.; LIOU, S.-J.; LAI, C.-Y.; WU, P.-C.; TSAI, T.-Y. 2001. Enhancement of Corrosion Protection Effect in Polyaniline via the Formation of PolyanilineClay Nanocomposite Materials. Chem. Materials. 13:1131-1136.

Recibido: Marzo 2 de 2012

Aceptado: Agosto 26 de 2012 